Research Journal of Applied Sciences 13 (7): 399-404, 2018

ISSN: $1815-932 \mathrm{X}$

(C) Medwell Journals, 2018

\title{
Design and Implementation of an Experimental Torsion Test Bench for Validation of Control Strategies
}

\author{
Mongui Daniel, Amaya Dario and Aviles Oscar \\ Nueva Granada Militar University, Bogota, Colombia
}

\begin{abstract}
The control theory is an area in continuous evolution, since, the classical control, passing through the modern control and ending in the intelligent control. It is the fundament of the modern industrial processes, aiming to improve the competitiveness and achieve high levels of productivity manufacturing organizations. This makes necessary the study of the control with an industrial view which is presented in different academic spaces where thematic contents are developed concerning the study of the systems dynamics, for their subsequent control. It is there where the support tool for laboratory practices reaches its greatest interest. In this project was designed and built an experimental torsion test bench, to validate different control strategies, specifically PID, sliding mode control and fuzzy control. This allows to make a comparative analysis between the proposed control structures, under different configurations of the proposed system.
\end{abstract}

Key words: Test bench, control, experimentation, magnetic disturbance, reference signal, structures

\section{INTRODUCTION}

Elaboration prototypes for a practical implementation involving mechanical, electronic, computational and control systems, concerns the mechatronic engineer, many test benches prototypes have been developed in different research works in which the knowledge is complemented with the practice (Tsai et al., 1997).

In many cases in the academic field, there has been a need to use systems with different characteristics that allow the integration of activities and knowledge in a practical way, since, the economic limitations in the acquisition of systems, coerce keeping knowledge in the theory or transfer knowledge through practical research and design (Asghari and Erfanian, 2017)

In fact if it is a content that has not been addressed in the classroom, by illustrating an experimental prototype using the teaching demonstration technique, the students can handle data and their interpretation of how it operates a physical principle to explain the observed phenomenon (Perez, 2008). Accordingly Rivero (2008) points out that demonstration experiments are necessary for students to realize that physics, for example, it is a natural science and that each theory is based on the responses that nature provides to the appropriate formulated questions, consequently in the same way this test bench can be used aiming this kind of practices. Then some examples of developed test benches are mentioned.

Luis et al. (2006) built an experimental bench that allows simulating real conditions of operation to evaluate and measure two photovoltaic pumping systems. Perez
(2009) present a set of experimental bench prototypes for the learning of optics. Zhang et al. (2011) developed hydraulic experimental banks for the teaching process to improve the effect of experimentation using a control system. Girin and Plestan (2009) present the design, modeling and control of an electro-pneumatic test bench.

This is how the design and development of test benches in different areas of knowledge allows real conditions to be simulated, to evaluate and measure system variables, to experimentally recreate theoretical concepts, to identify and verify designed control techniques, allowing the analysis and evaluation of performance in each test. The use of technologies and the possibility of new pedagogical-didactic approaches lead to corroborate the theory through physical and practical implementations that allow to perceive the real applications of the different branches of the theoretical knowledge.

In this development, the main idea was the comparison between the performance of PID control (Marisol, 2013) Sliding Mode Control (SMC) and fuzzy control (Khatun et al., 2003; Cabrera et al., 2005) analyzing the results in a physical application that allows to study the behavior of a test bench. The test bench allows to test different control methods and torsional behavior with external disturbances, what allow teaching the students how to work in the laboratory and train them for situation in engineering and applied sciences. The techno-pedagogy is revolutionizing the traditional education by bringing the laboratory experience with the theoretical formation simultaneously.

Corresponding Author: Mongui Daniel, Nueva Granada Militar University, Bogota, Colombia 


\section{MATERIALS AND METHODS}

Test bench development: Consists of two disks connected by an interchangeable longitudinal element (torsion bar) which is in a vertical position, the disks act as the perturbation of the dynamical system to be affected by the magnetic field of the coils (Fig. 1).

Mechanical design of the test bench: The test bench was designed in SolidWorks, through the CAD. Later was developed physically considering the best functioning, stability, manipulation and aesthetics of the prototype (Fig. 2).

In the lower part, the bar is rigidly coupled to a gearmotor with direct current encoder (37DX57L); the upper part, it is rigidly coupled to an encoder (E50S8) which verifies the speed respect to the lower one. The bench has two magnetic brakes, the discs that are directly and independently affected by two coils when is applied a potential difference they generate a magnetic field, consequently, through the relative movement between the disc and the coils.

A current (eddy current) or electron flow is induced inside the conductor, creating a magnetic field that opposed to the generated by the coils, (Fig. 3). For powering the gearmotor with encoder, the encoder and the coils, a power supply circuit was designed with two power sources where the circuit is based on the L298N chip, what contains optocoupled inputs for module control.

The control module is from the arduino PWM with an interface for feed the encoders and for the train pulse conditioning of the speed sensor output which is recorded by the arduino timerl and timero which the function is the measure the frequency. To achieve the close loop control, a controller design was created in Simulink and it executed from it. A feedback circuit was used and a second order PID was used in the transfer function to generate the PWM output for the Arduino. The program uses the timer 3 interrupt service every 1.5 milliseconds, takes the pulses recorded from the timer 5 and timer 0 which match to each encoder as shown in Fig. 4.

\section{Controllers}

PID control: The transfer function in the $\mathrm{z}$ transform domain, to proceed to calculate the discrete PID controller is given by Eq. 1:

$$
G(z)=\frac{0.5976 z+4.2765 \times 10^{-4}}{z^{2}-0.3623 z}
$$

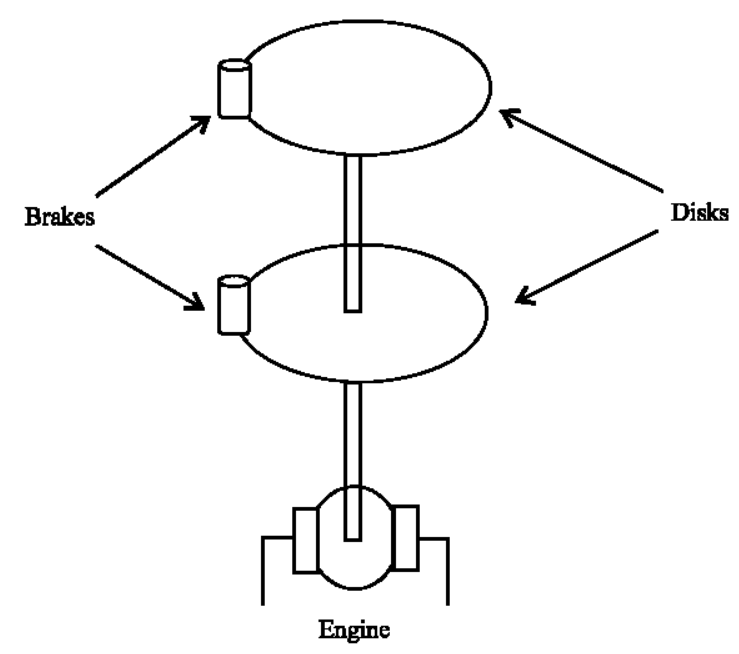

Fig. 1: Overview of the test bench

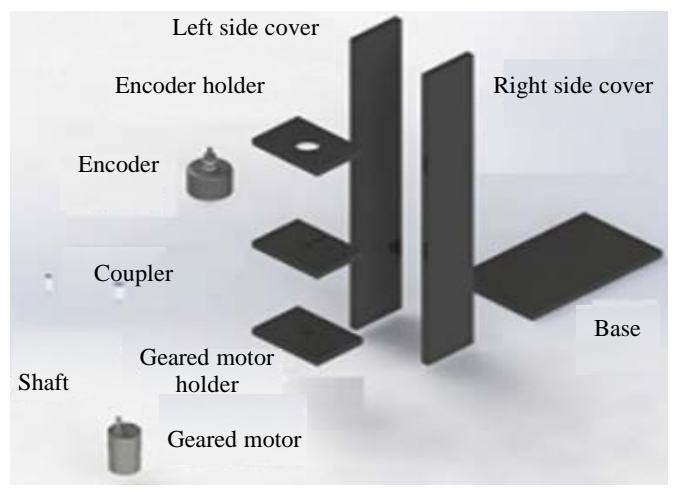

Fig. 2: Exploded view of the bench

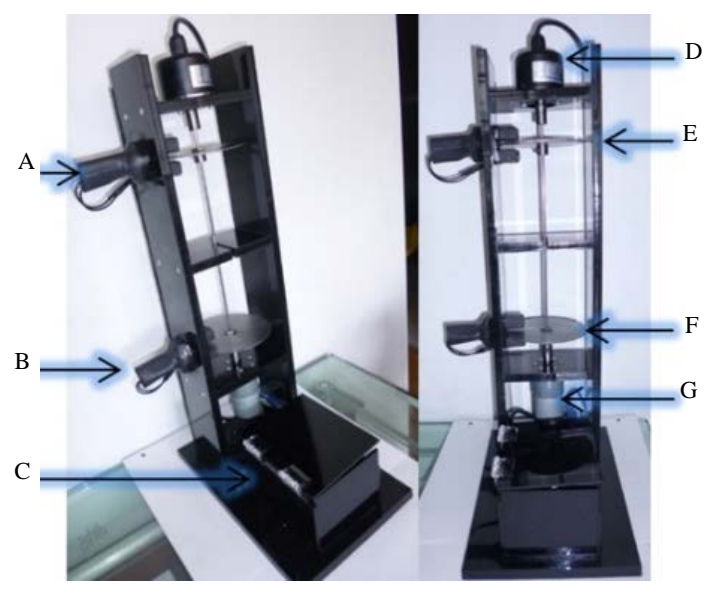

Fig. 3: Physical bench and its parts: A) Upper magnetic brake; B) Lower magnetic brake; C) Circuit box; D) Encoder; E) Upper disc; F) Lower disc and G) Gearmotor 
Sliding mode control: For the bench motor parameters shown in Table 1, the numerically designed controller is given by the Eq. 2 and 3 :

$$
\begin{gathered}
\sigma=0.08967 \times 10^{-4}(\omega-\mathrm{r})+0.09261 \times 10^{-6} \omega \\
\mathrm{U}=\left(0.08967 \times 10^{-6}\right)(35861.1 \omega-3586188.9(\omega-\mathrm{r})+ \\
7501.0167 \sigma-\operatorname{sgn}(\sigma))
\end{gathered}
$$

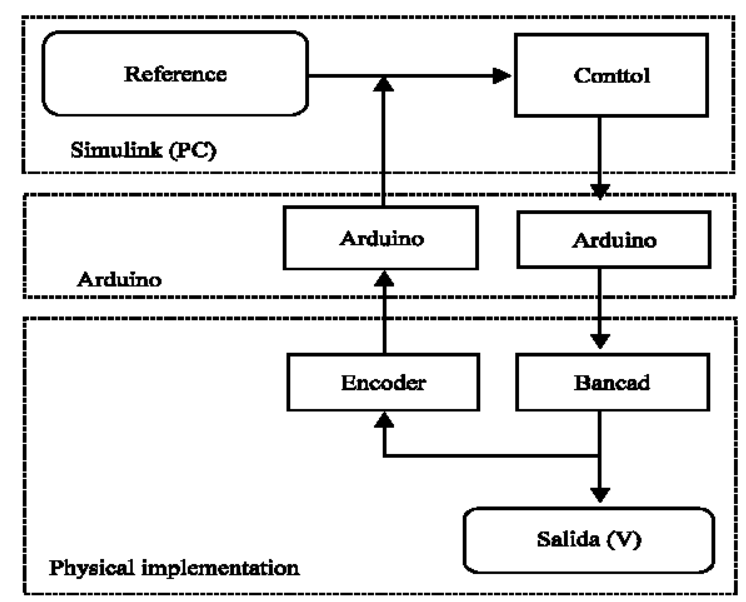

Fig. 4: Communication flow diagram
Motor parameters: The values in Table 1 were adjusted from those obtained by Marisol (2013) where the same motor is used with those of a Maxon DC motor, looking for the best approximation.

Fuzzy control: There are two input variables, the motor speed and the error. The motor speed has 5 membership functions: High speed, high medium speed, constant speed, low medium speed and low speed as shown in Fig. 5.

The error variable has two membership functions: high negative error and high positive error as shown in Fig. 6. Finally, the output of the control is the voltage for which there are also five membership functions; greatly decreased, little decreased, hold, little increased and greatly increased as shown in Fig. 7.

\section{Table 1: Motor parameters}

\begin{tabular}{ll}
\hline Parameters & Values \\
Ke & $0.290 \mathrm{Vs}$ \\
$\mathrm{Km}$ & $46.0 \times 10^{-3} \mathrm{NmA}^{-1}$ \\
$\mathrm{~J}$ & $4.431 \times 10^{6} \mathrm{~kg}^{2}$ \\
$\mathrm{R}$ & $7.180 \Omega$ \\
$\mathrm{L}$ & $0.9540 \times 10^{-3} \mathrm{H}$ \\
$\mathrm{b}$ & $2.98 \times 10^{-4} \mathrm{Nm} \mathrm{sec}$ \\
$\lambda$ & -101.0 \\
$\mathrm{Ks}$ & 1 \\
$\mathrm{Kp}$ & 0 \\
\hline
\end{tabular}

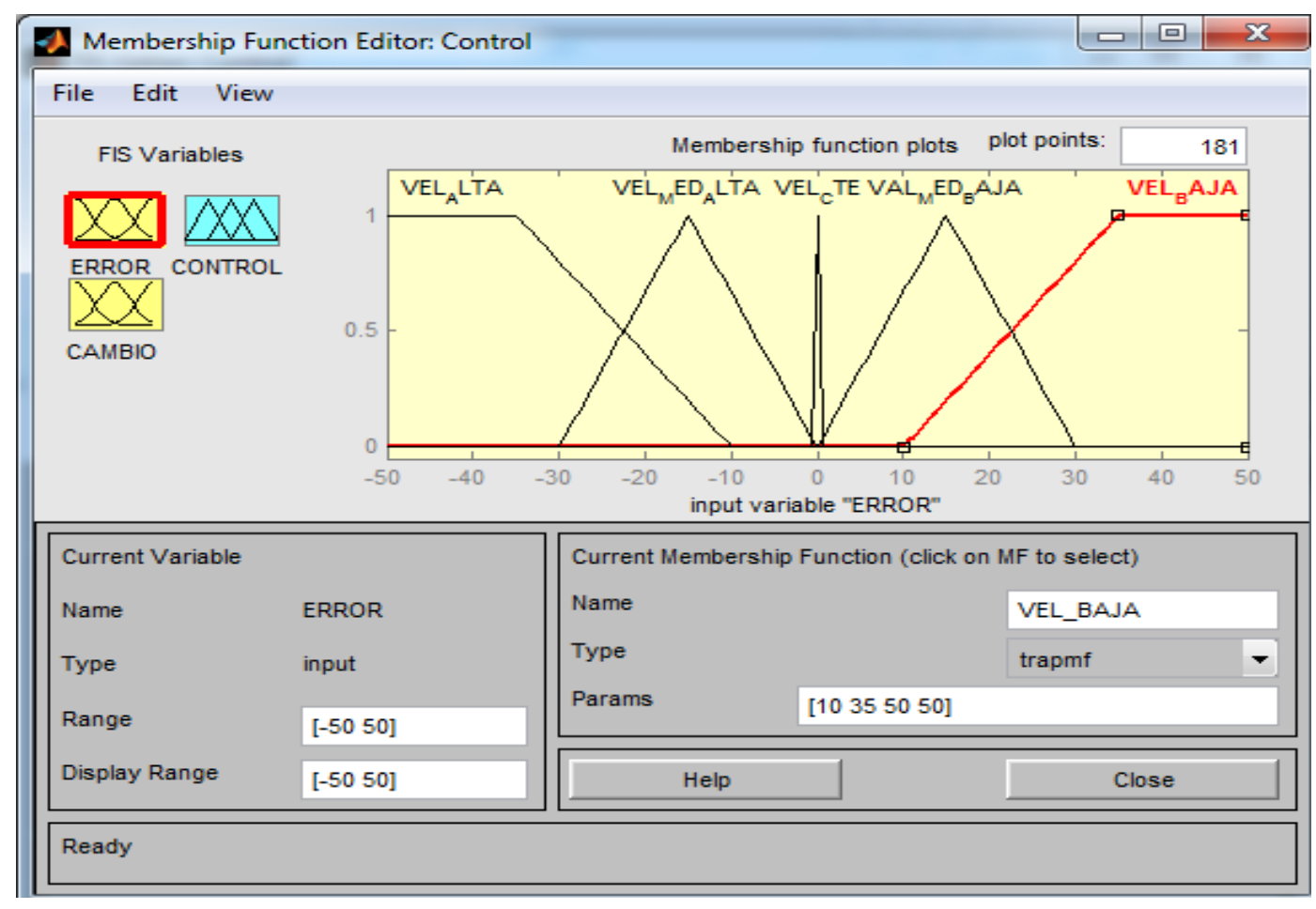

Fig. 5: Motor speed membership functions 
Res. J. Applied Sci., 13 (7): 399-404, 2018

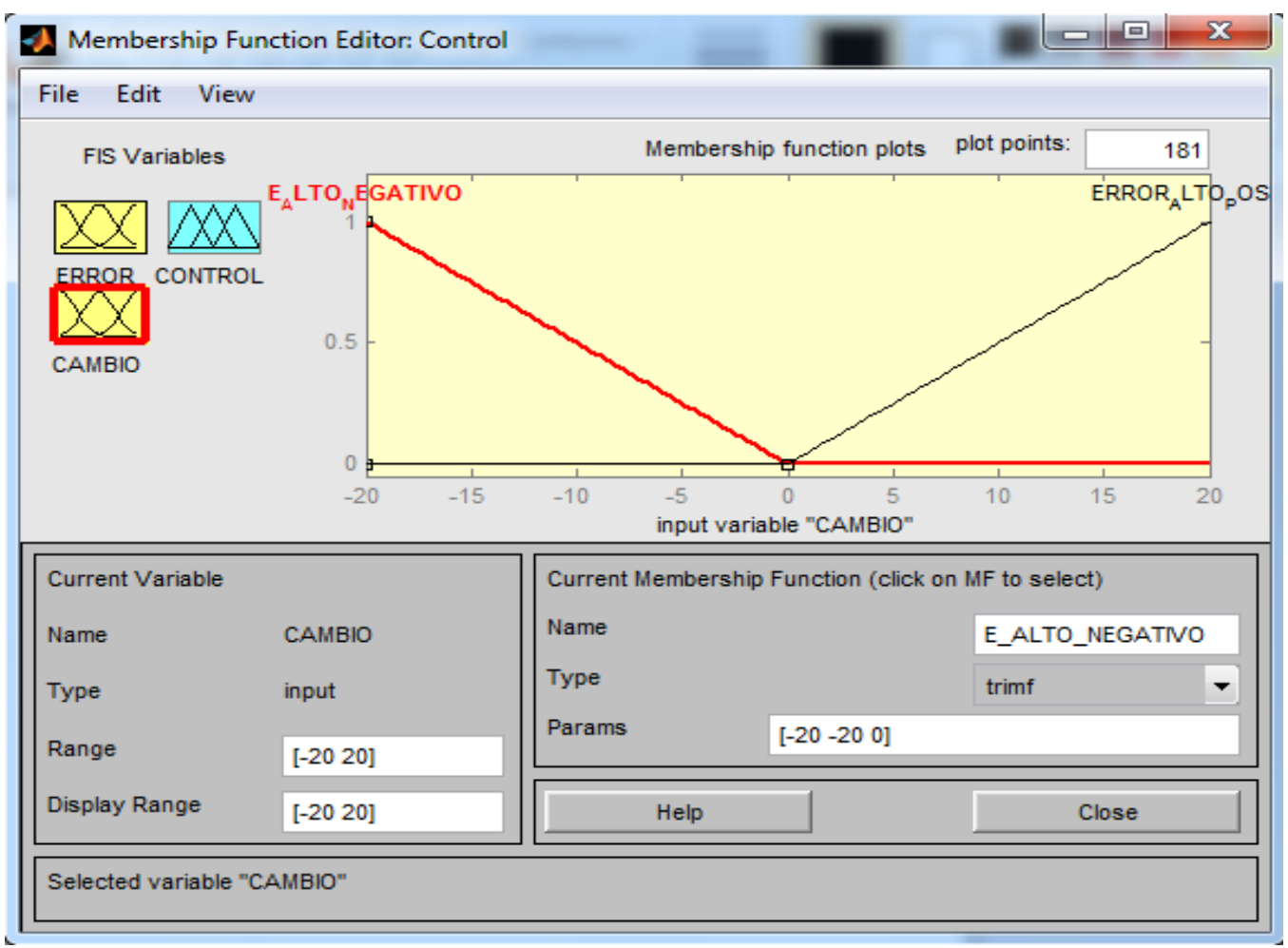

Fig. 6: Error membership functions

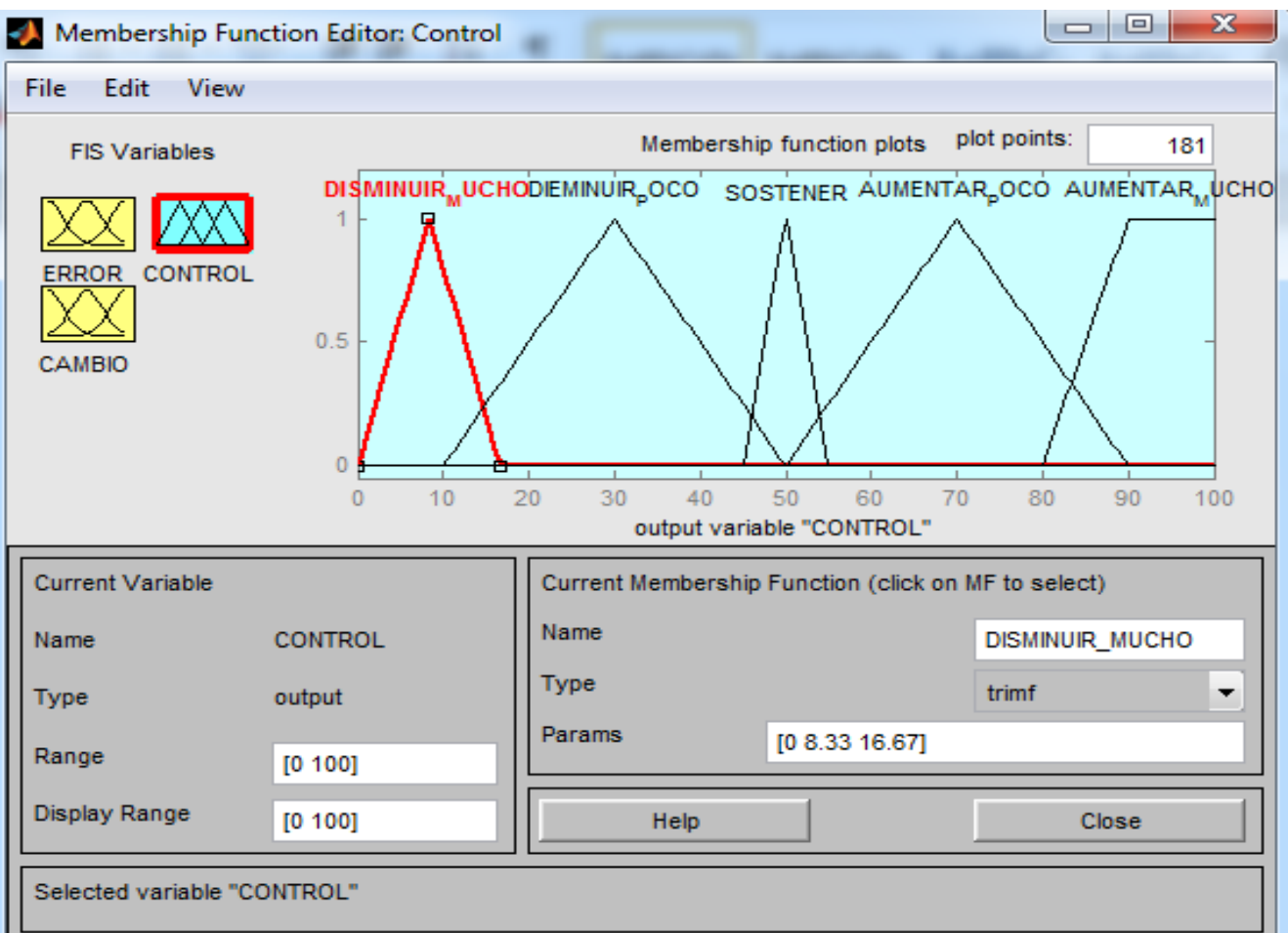

Fig. 7: Control membership functions (voltage) 
The fuzzy rules as implicit representation of the model, they can deduce the behavior of the system, considering two antecedents and a consequent. These are listed.

- If error is low speed, then control is greatly increased

- If error is high speed, then control is greatly decreased

- If error is constant speed and change is high negative error, then control is Little decreased

- If error is constant speed and change is positive high error, then control is to increase little

- If error is medium high speed, then control is little decreased

- If error is low medium speed, then control is to Little increase

- If error is constant speed, then control is to hold

\section{RESULTS AND DISCUSSION}

The experimental results obtained for the closed loop system at nominal load for the PID control is observed in Fig. 8. The system output quickly reaches the reference value which shows the appropriate calibration of the devices (actuator and sensor).

Figure 9 shows how the performance of the system is affected by the magnetic braking effect, indicated by the ellipses in the system response. After including the PID control action, the result of the behavior of the controlled system is shown under the action of the disturbance, showing a great capacity of the system to maintain the reference value in values above the average of speed.

In Fig. 10 is shows the behavior of the control signal by sliding mode control for different reference values where is possible to perceive the average constant value of the control signal during the time interval in the same way the chattering around the reference signal is shown.

In Fig. 11, the magnetic disturbance indicated with ellipses is evidenced as well the robustness of the sliding modes control technique implemented for which a significant increase in the control effort is obtained by adding the perturbation as the Fig. 10 shows where the chattering around the reference signal is perceived.

Figure 12, shown that the speed is regulated for different reference values with the fuzzy logic technique implemented for the speed control of the plant. Both above and below of the average value of set point is evidenced how the desired value is reached.

In Fig. 13, the magnetic disturbance is evidenced, indicated by the ellipses, after being activated in the test bench. With the fuzzy logic

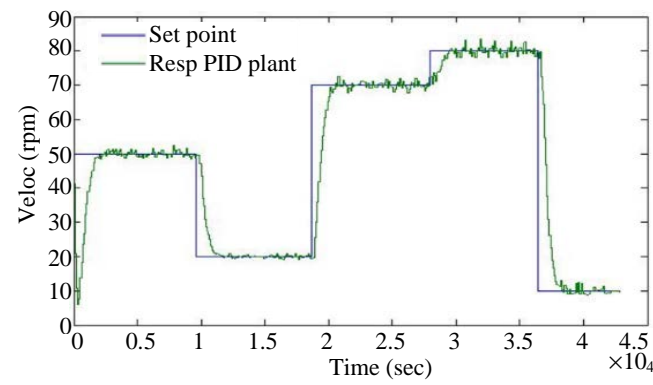

Fig. 8: Response of the plant using a PID controller

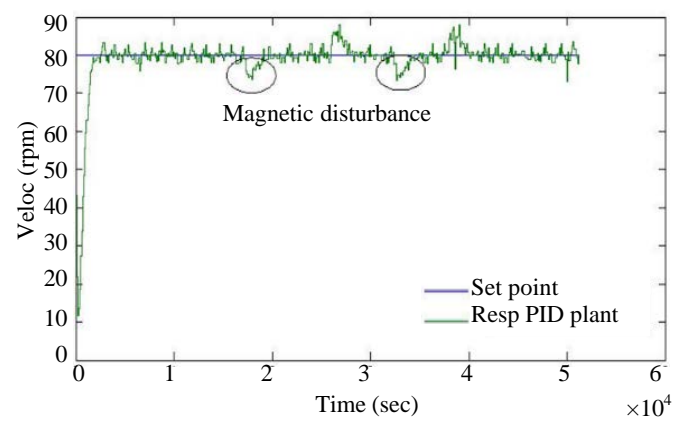

Fig. 9: Response of the plant using a PID controller under magnetic disturbance

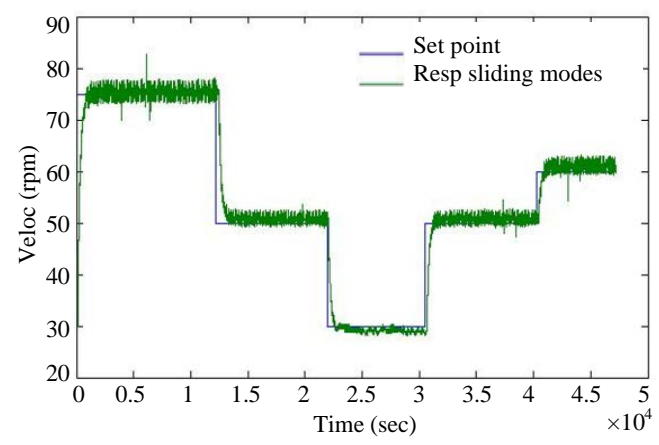

Fig. 10: Response of the plant using sliding mode control

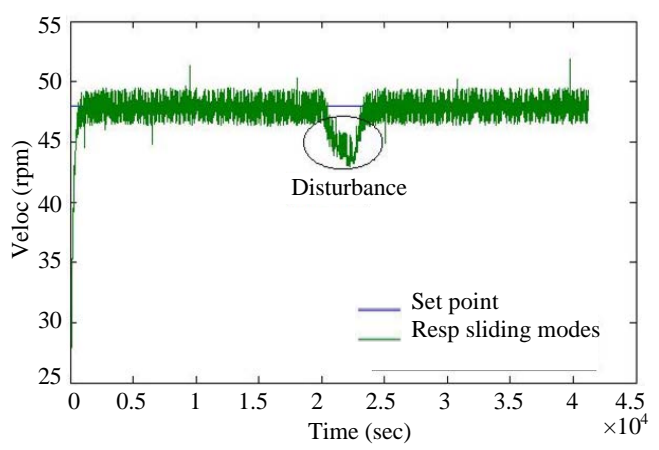

Fig. 11: Response of the plant using sliding mode control under magnetic perturbation 


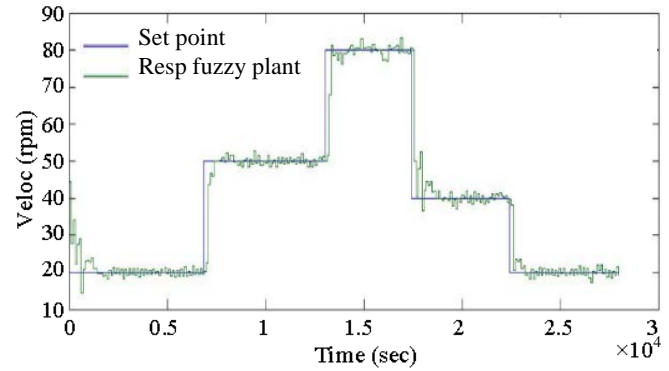

Fig. 12: Response of the plant using a fuzzy logic controller

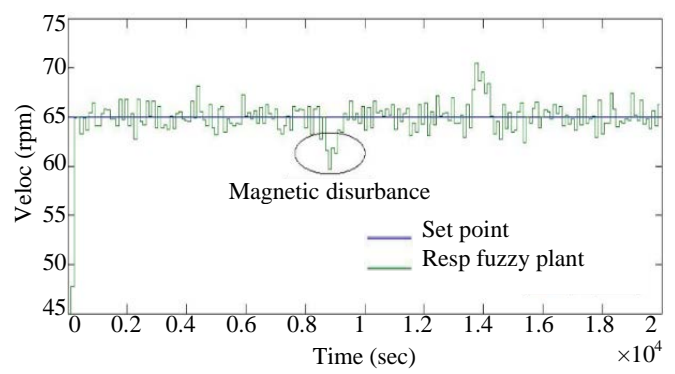

Fig. 13: Response of the plant using a fuzzy logic controller under magnetic perturbation

technique, the system returns to its reference value, so, the proposed inference rules perform a satisfactory speed recovery.

\section{CONCLUSION}

The design and physical development of a practical torsional type bench of easy manipulation with great stability, versatility and attractive for the students of the area where are included the variable angular velocity with perturbations of magnetic braking to the system was successfully achieved.

A comparative analysis of the performance of the validated control techniques in the physical plant was carried out and it was concluded that the controllers designed by sliding mode control and fuzzy logic present a superior performance than the PID technique in the tracing of reference values or set point of speed, under external load disturbances.

Although, the SMC controller presents the problem of chattering or high frequency oscillation around the desired value and it requires a significant increase in the control signal, it is evidenced that it is more robust than the other two types of control, it is less sensitive to disturbances, it gives a faster response to the system, making it more efficient and with better performance.

\section{ACKNOWLEDGEMENT}

This research was supported by the GAV and DAVINCI Researchs Groups of Militar Nueva Granada University in Bogota-Colombia

\section{REFERENCES}

Asghari, M.A.S.L. and A.R. Erfanian, 2017. Designing and modeling of an infrared scene generator using SMD resistor arrays for use in the simulator of hardware in the loop. J. Eng. Appl. Sci., 12: 1050-1059.

Cabrera, J.A., A. Ortiz, J.J. Castillo and A. Simon, 2005. A fuzzy logic control for antilock braking system integrated in the IMMa tire test bench. IEEE. Trans. Veh. Technol., 54: 1937-1949.

Girin, A. and F. Plestan, 2009. A new experimental test bench for a high performance double electropneumatic actuator system. Proceedings of the Conference on American Control (ACC'09), June 10-12, 2009, IEEE, St. Louis, Missouri, USA., ISBN:978-1-4244-4523-3, pp: 3488-3493.

Khatun, P., C.M. Bingham, N. Schofield and P.H. Mellor, 2003. Application of fuzzy control algorithms for electric vehicle antilock braking/traction control systems. IEEE. Trans. Veh. Technol., 52: 1356-1364.

Luis, V.H., M.A. Steigleder, M.R. Aeberhard and F. Benitez, 2006. [Test bench to determine the performance of low power SBFV]. Master Thesis, Faculty of Engineering, National University of the Northeast, Corrientes, Argentina.

Marisol, A., 2013. [Design, construction and control of a The double inverted rotational pendulum]. Master Thesis, National Autonomous University of Mexico, Mexico City, Mexico. (In Spanish)

Perez, E., 2008. [Design of experimental models and prototypes oriented to the learning of the optics]. Ph.D Thesis, University of Carabobo, Valencia, Venezuela. (In Spanish)

Tsai, M.C., C.C. Chou and M.F. Hsieh, 1997. Development of a real-time servo control test bench. IEEE. Trans. Educ., 40: 242-252.

Zhang, C., X. Wang and Z. Wang, 2011. Design of the control system for hydraulic experimental bench based on LabVIEW. Proceedings of the 2011 International Conference on Electronic and Mechanical Engineering and Information Technology (EMEIT) Vol. 4, August 12-14, 2011, IEEE, Harbin, China, ISBN:978-1-61284-087-1, pp: 2128-2130. 